\title{
Heavy-quark momentum correlations as a sensitive probe of thermalization
}

\author{
K. Schweda and G. Tsiledakis \\ Physikalisches Institut, Universität Heidelberg, D-69120 Heidelberg, Germany
}

\begin{abstract}
In high-energy nuclear collisions the degree of thermalization at the partonic level is a key issue. Due to their large mass, heavy-quarks and their participation in the collective flow of the QCD medium constitute a powerful tool to probe thermalization. We propose measuring azimuthal correlations of heavy-quark hadrons and products from their semi-leptonic decay. Modifications or even the complete absence of initially, e.g. in $p p$ collisions, existing azimuthal correlations in $\mathrm{Pb}+\mathrm{Pb}$ collisions might indicate thermalization at the partonic level. We present studies with PYTHIA for $p p$ collisions at the top LHC energy using the two-particle transverse momentum correlator $\left\langle\bar{\Delta} p_{t, 1} \bar{\Delta} p_{t, 2}\right\rangle$ as a sensitive measure of azimuthal correlations.
\end{abstract}

\section{Introduction}

High-energy nuclear collisions offer the unique opportunity to probe highly excited nuclear matter in the laboratory. At sufficiently high temperature and/or energy density hadrons dissolve and quarks and gluons carrying color charge are the relevant degrees of freedom, commonly called a Quark Gluon Plasma (QGP). An essential difference between collisions of elementary particles on the one hand and heavy nuclei on the other hand is the development of collectivity in the latter. Collective flow of hadrons, especially the multi-strange hadrons $\phi$ and $\Omega$, has been experimentally observed at RHIC [1] suggesting that collectivity dominantly develops in the early partonic stage, i.e. among quarks and gluons. Presently, the degree of thermalization among partons remains a crucial issue.

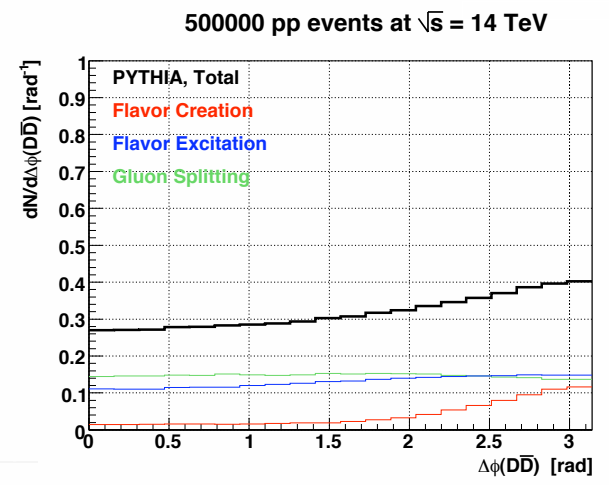

Figure 1. (Color online) Distribution of $D \bar{D}$ pairs as a function of relative azimuth $\Delta \phi$ from $p p$ collisions at $\sqrt{s}=14 \mathrm{TeV}$ as calculated by PYTHIA (v. 6.406) integrated over rapidity from flavor creation (red line), flavor excitation (blue line), gluon splitting (green line), and their sum (black line). 
Heavy-quark hadrons and their observables are of particular interest when addressing thermalization 2. In a QGP, where chiral symmetry might be partially restored and consequently light-quarks (up, down, strange) obtain their small bare masses, heavy-quarks remain massive [3. Heavy-quarks are mostly created in the early stage of the collision, since their mass is much larger than the maximum possible temperature of the QCD medium created while annihilation in the QCD medium is negligible [4. Thus, heavy-quarks probe the entire history of a high-energy nuclear collision. Heavy-quarks participate in collective motion provided their interactions at the partonic level occur frequently. Thus, collective motion of heavy-quark hadrons is a powerful tool when addressing early thermalization of light quarks in high-energy nuclear collisions.

\section{1. $D \bar{D}$ angular correlations}

In strong interactions heavy-quarks are always created together with their anti-quark and are thus correlated. In collisions of elementary particles, these correlations survive the fragmentation process into hadrons to a large extent [3] and are thus observable e.g. in the angular distributions of pairs of $D$ - and $\bar{D}$-mesons [5].

In high-energy collisons of heavy nuclei, frequent interactions among partons (quarks and gluons) of the QCD medium and heavy-quarks would lead to a weakening and finally complete vanishing of these correlations. On the other hand, hadronic interactions at the late stage are insufficient to alter azimuthal correlation of $D \bar{D}-$ pairs [3. It is frequent interactions distributing and randomizing the available (kinetic) energy and finally driving the system, i.e. light-quarks and gluons, to local thermal equilibrium. Thus, the decreasing magnitude when compared to $p p$ collisions of heavy-quark correlations in high-energy collisons of heavy nuclei would indicate early thermalization among partons.

We employed the Monte Carlo event generator PYTHIA [6] which reproduces experimentally observed correlations of $D$ mesons at fixed target energies [5]. Parameters in PYTHIA were tuned to reproduce the NLO predictions [7, 8. Our results on the yield of $D \bar{D}$-pairs versus their relative azimuth for $p p$ collisions at the top LHC energy of $\sqrt{s}=14 \mathrm{TeV}$ are shown in Fig. 1. Our calculations at leading order (LO) contain flavor creation processes $(q \bar{q} \rightarrow Q \bar{Q}, g g \rightarrow Q \bar{Q})$ and lead to an enhancement around $\Delta \phi \approx 180^{\circ}$, i.e. back-to-back. However, next-toleading order (NLO) contributions such as flavor excitation $(q Q \rightarrow q Q, g Q \rightarrow g Q)$ and gluon splitting $(g \rightarrow Q \bar{Q})$ become dominant at LHC energies and do not show pronounced correlations in the representation chosen in Fig. 1 leading to a rather weak dependence on relative azimuth. By choosing various momentum cuts, a somewhat stronger correlation was extracted [9].

In this paper, we introduce the two-particle transverse momentum correlator as a sensitive measure of heavy-quark correlations. This method has the following advantages:

(i) the correlator is sensitive to non-statistical fluctuations, thus carving out any physical correlations and eliminating the need for various momentum-cuts in the analysis. This is essential when comparing results from different collision systems, e.g. $p p$ and $\mathrm{Pb}+\mathrm{Pb}$.

(ii) in case of physically uncorrelated candidate-pairs (e.g. background), the extracted value for the correlator is null, thus providing a reliable baseline. 


\section{Employing the two-particle transverse momentum correlator}

The occurence of non-statistical fluctuations of the event-by-event mean transverse momentum $M_{p t}$ goes along with correlations among the transverse momenta of particle pairs. Such correlations were successfully extracted from experimental data employing the two-particle transverse momentum correlator [10, 11]. For $D$ - and $\bar{D}-$ mesons, this leads to

$$
\left\langle\Delta p_{t, 1}, \Delta p_{t, 2}\right\rangle^{(D \bar{D})}=\frac{\sum_{k=1}^{n_{\mathrm{ev}}} C_{k}}{\sum_{k=1}^{n_{\mathrm{ev}}} N_{k}^{\text {pairs }}}
$$

where $C_{k}$ is the $p_{t}$ convariance:

$$
C_{k}=\sum_{i=1}^{N_{k}} \sum_{j=1}^{N_{k}}\left(p_{t i}-\bar{p}_{t}^{(D)}\right)\left(p_{t j}-\bar{p}_{t}^{(\bar{D})}\right)
$$

where $p_{t i}$ and $p_{t j}$ are the transverse momentum $p_{t}$ for $i^{t h}$ and $j^{t h}$ particle of an event of $D$ - and $\bar{D}$-mesons respectively, $\overline{p_{t}}$ is the inclusive mean transverse momentum averaged over all particles of all events of $D$ and $\bar{D}, \sum_{k=1}^{n_{\mathrm{ev}}} N_{k}^{\text {pairs }}$ the total number of $D \bar{D}$ pairs and $n_{e v}$ the total number of $p p$ collisions.

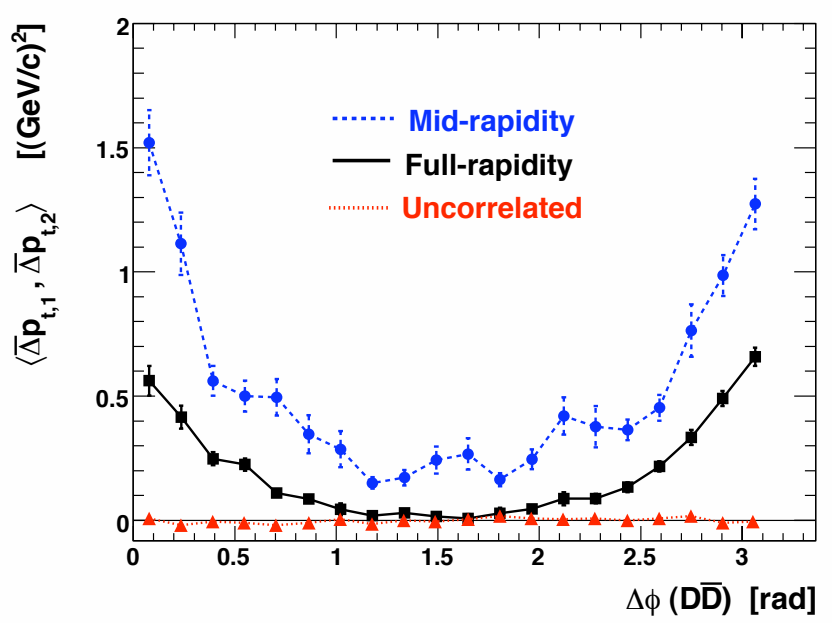

Figure 2. (Color online) Distribution of the momentum correlator $\left\langle\Delta p_{t, 1}, \Delta p_{t, 2}\right\rangle$ of $D \bar{D}$ pairs as a function of relative azimuth $\Delta \phi$ at mid-rapidity (circles), integrated over rapidity (squares) and for background using the mixed event method (triangles) from $p p$ collisions at $\sqrt{s}=14 \mathrm{TeV}$ as calculated by PYTHIA (v. 6.406). The lines are drawn to guide the eye.

We studied the scale dependence of $p_{t}$ correlations in azimuthal space by calculating the correlator in bins of the relative azimuthal separation $\Delta \phi$ of particle pairs. For the case of independent particle emission from a single parent distribution, the correlator $\left\langle\Delta p_{t, 1}, \Delta p_{t, 2}\right\rangle$ vanishes.

The $D \bar{D}$ momentum correlator $\left\langle\Delta p_{t, 1}, \Delta p_{t, 2}\right\rangle$ as a function of relative azimuth $\Delta \phi$ is shown in Fig. 2 for $p p$ collisions at $\sqrt{s}=14 \mathrm{TeV}$ exhibiting a rich structure. We observe an enhancement at small azimuth from gluon splitting processes, while flavor creation of $c \bar{c}$-quark pairs leads to a pronounced distribution at backward angels. 

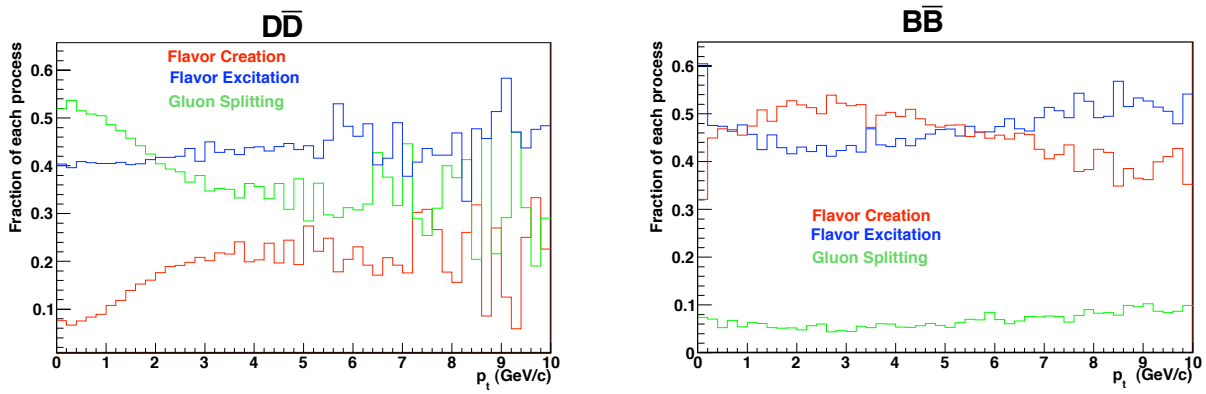

Figure 3. (Color online) Relative contribution to the production of $D$ - and $\bar{D}-$ mesons (left) and $B$ - and $\bar{B}$-mesons (right) as a function of transverse momentum for the processes of flavor creation (red line), flavor excitation (blue line) and gluon splitting (green line) from $p-p$ collisions at $\sqrt{s}=14 \mathrm{TeV}$ as calculated by PYTHIA (v. 6.406).

Flavor excitation processes involve a larger number of gluons leading to a rather flat distribution At mid-rapidity, the correlations are even stronger, reflecting the harder $p_{t}$ spectrum of $D \bar{D}$ meson-pairs when compared to the full rapidity range. Integrating the correlator over all azimuth, we get $\left\langle\Delta p_{t, 1}, \Delta p_{t, 2}\right\rangle=0.199 \pm 0.006 \mathrm{GeV}^{2} / c^{2}$ which corresponds to the normalized dynamical fluctuation $\Sigma_{p t}[12$ of $\sim 30 \%$ in $\overline{p_{t}}$. This large value implies a strong correlation when compared to $\sim 1 \%$ observed for unidentified charged particles in central collisions at SPS and RHIC [12, 13, 14]. To mimic combinatorial background which is always present in the experiment, we applied the correlator to $D$ - and $\bar{D}$ - mesons from different $p p$ collisions, which are physically uncorrelated. This results in a value of $\left\langle\Delta p_{t, 1}, \Delta p_{t, 2}\right\rangle$ consistent with zero. Therefore the correlator allows for a clear distinction between the case were correlations are present (different from zero) or absent (equal to zero) in contrast to the method described in 3 . The different QCD processes contributing to heavy-quark productions have strong energy dependences. Figure 3 shows the relative contribution to the production of $D$ - and $\bar{D}$-mesons (left) and $B$ - and $\bar{B}$-mesons (right) as a function of transverse momentum. The production of $D$-mesons is dominated by gluon splitting and flavor excitation processes while the contribution from flavor creation is about $10 \%$ at low momentum and increases up to $20 \%$ at larger momentum. At RHIC energies, the contribution from gluon splitting was estimated by the STAR experiment to be less than $10 \%$ [15]. On the other hand, the production of $B$-mesons is dominated by flavor creation and flavor excitation with a small contribution from gluon splitting below $10 \%$ and an overall weak dependence on transverse momentum.

As shown above, the initial correlations of $c \bar{c}$-quark pairs survive the fragmentation process to a large extent. However experimentally, full kinematic reconstruction of $D$-mesons from topological decays suffer from small efficiencies resulting in low statistics, especially when pairs of $D$-mesons are considered where the reconstruction efficiency enters quadratically. To circumvent this fact, we considered electrons (positrons) from semi-leptonic decays of $D$ - and $B$-mesons with a branching ratio to electrons of $10 \%$ and $11 \%$, respectively.

Figure 4 shows the momentum correlator $\left\langle\Delta p_{t, 1}, \Delta p_{t, 2}\right\rangle$ of $e^{+} e^{-}$-pairs from decays of $D \bar{D}$ (squares) and $B \bar{B}$-mesons pairs (triangles) as a function of their relative azimuth $\Delta \phi$ integrated over the full rapidity range. We chose a momentum cut 


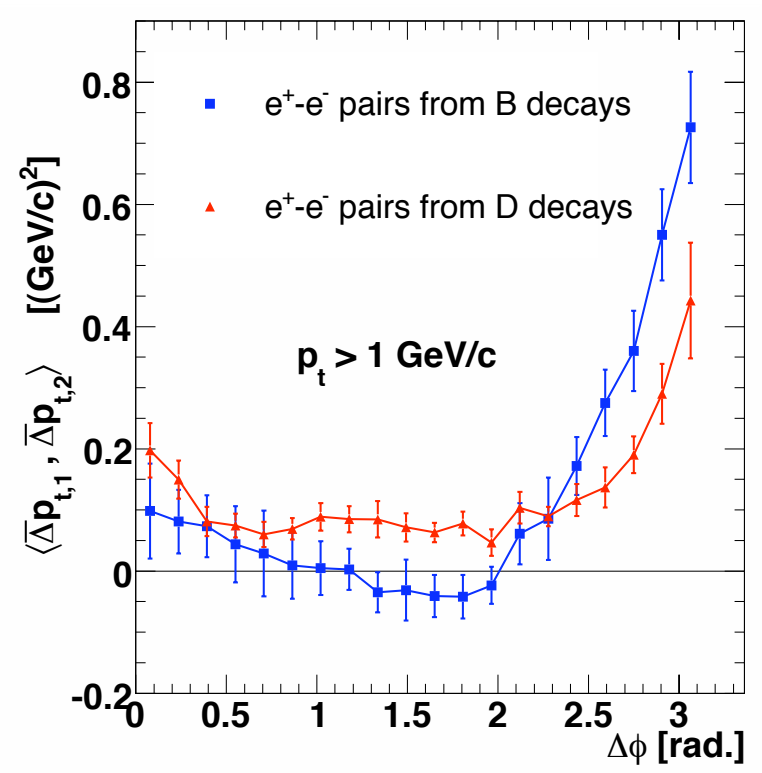

Figure 4. (Color online) Distribution of the momentum correlator $\left\langle\Delta p_{t, 1}, \Delta p_{t, 2}\right\rangle$ of $e^{+} e^{-}$pairs stemming from semi-leptonic decays of pairs of $B \bar{B}-$ mesons (squares) and $D \bar{D}$-mesons (triangles) as a function of relative azimuth $\Delta \phi$ at mid-rapidity in $p p$ collisions at $\sqrt{s}=14 \mathrm{TeV}$ as calculated by PYTHIA (v. 6.406). The lines are drawn to guide the eye.

of $p_{t}>1 \mathrm{GeV} / c$ allowing for clean electron identification at reasonable background and still sufficient statistics in the ALICE experiment using the Transition Radiation Detector. We observe a correlation at forward angles due to gluon splitting and a more pronounced correlation at away-side angles from flavor creation processes indicating that the initial correlations among heavy-quarks and their anti-quark even survive the semi-leptonic decay into electrons (positrons) making an experimental observation at the LHC possible. We note that the correlation is stronger for electrons from semileptonic decays of $B$-mesons compared to $D$-mesons. This is in agreement with results from the STAR experiment at RHIC [16.

When integrating over full azimuth for $D$-mesons, we get a value for the correlator of $\left\langle\Delta p_{t, 1}, \Delta p_{t, 2}\right\rangle=0.007 \pm 0.001 \mathrm{GeV}^{2} / c^{2}$, which corresponds to the normalized dynamical fluctuation $\Sigma_{p t}$ of $\sim 12 \%$. Hence, more than half of the correlation strength observed for fully reconstructed $D$-meson pairs is still present in pairs of electrons and positrons from semi-leptonic decays.

\section{Conclusions and outlook}

In summary, we propose measuring correlations of heavy-quarks and their modifications in $\mathrm{Pb}+\mathrm{Pb}$ collisions compared to $p p$ collisions as a sensitive probe of thermalization at the early partonic stage. We applied the momentum correlator as a precise and normalized measure. Different QCD processes contributing to heavyquark production are identified in the azimuthal distribution of the correlator. Our 
results indicate that these correlations born at the creation of pairs of heavy-quarks and their anti-quarks survive the fragmentation in $p p$ collisions into hadrons and even semi-leptonic decays into electrons to a large extent. Thus, experimental observation of these correlations in $p p$ and $\mathrm{Pb}+\mathrm{Pb}$ collisions at the LHC seems feasible.

\section{Acknowledgment}

We would like to thank Drs. Y. Pachmayer and N. Xu for exciting discussions. This work has been supported by the Helmholtz Association under contract number VHNG-147.

[1] J. Adams et al. (STAR collaboration), Phys. Rev. Lett. 95, 122301 (2005);

B.I. Abelev et al. (STAR collaboration), Phys. Rev. Lett. 99, 112301 (2007).

[2] O. Linnyk, E.L. Bratkovskaya, and W. Cassing, Int. J. Mod. Phys. E17, 1367 (2008).

[3] X. Zhu et al., Phys. Lett. B 647, 366 (2007); X. Zhu, these proceedings.

[4] P. Braun-Munzinger, J. Phys. G 34 S471 (2007).

[5] C. Lourenço and H.K. Wöhri, Phys. Rept. 433, 127 (2006).

[6] T. Sjöstrand et al., Comput. Phys. Commun 135, 238 (2001).

[7] E. Norrbin and T. Sjöstrand, Eur. Phys. J. C 17, 137 (2000).

[8] N. Carrer and A. Dainese (ALICE Collaboration), arXiv:hep-ph/0311225

[9] X. Zhu et al., Phys. Rev. Lett. 100, 152301 (2008).

[10] J. Adams et al. (STAR collaboration), Phys. Rev. C 72, 044902 (2005).

[11] D. Adamova et al. (CERES collaboration), Nucl. Phys. A 811, 179 (2008).

[12] D. Adamova et al. (CERES collaboration), Nucl. Phys. A 727, 97 (2003).

[13] H. Sako et al. (CERES collaboration), J. Phys. G 30, S1371 (2004).

[14] M. Rybczynski et al. (NA49 collaboration), J. Phys. Conf. Ser. 5, 74 (2005).

[15] A. Mischke et al. (STAR collaboration), J. Phys. G 35, 104117 (2008).

[16] X. Lin et al. (STAR collaboration), Nucl. Phys A 783497 (2007). 